\title{
Thermopower Monitoring of Relaxation and Devitrification in Fe-Based Glassy Alloys
}

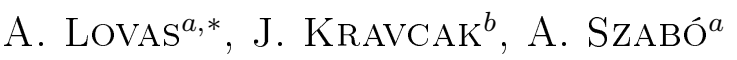 \\ ${ }^{a}$ Budapest University of Technology and Economics, Department of Automibiles and Vehicle Manufacturing, \\ Budapest H-1111, Bertalan Lajos utca 2. Hungary \\ ${ }^{b}$ Technical University of Košice, Department of Physics, , Park Komenského 2, 04200 Košice, Slovakia
}

The change of thermopower is investigated in Fe-based glassy alloys during structural relaxation and amorphous-crystalline transformation. It was found, that thermopower shifts to negative direction until solely the relaxation proceeds. This negative shift is composition independent. The net shape of thermopower curves is composed from the thermopower of the individual constituent crystalline phases after the crystallization process.

DOI: 10.12693/APhysPolA.126.78

PACS: 61.43.Dq 72.15.Jf

\section{Introduction}

Though the Seebeck effect was originally defined as the

$$
S_{A B}=\int_{T_{1}}^{T_{2}}\left(S_{B}-S_{A}\right) d T
$$

difference of potential between two contacting bulk, pure metal surfaces, recently it is also applied for stress detection or for the study of phase transformations in alloy systems $[1,2]$. In spite of the wide range of applications, the understanding of the key factors in the evaluation of $\mathrm{S}$ is not yet complete. Due to their single phase nature, the glassy alloys represent attractive model alloys for the separation of the mentioned factors. An additional advantage is the considerable stress accumulation in these materials, arising either from chemical reactions [3], or from the preparation technique itself [4]. When stress relieve occurs, (structural relaxation at low temperature) it can be separately studied either by magnetic method or by thermopower measurements. Hence, the role of stress state and the role of crystallization (phase transformation) can be studied independently. This is the purpose of the present paper.

\section{Experimental, materials and measurement methods, heat treatments}

The measurements were performed on Fe-B, and FeNi-B glassy samples, because of their well known thermal stability, and the previous study of their stress state $[5,6]$. Isothermal heat treatments were carried out, resulting in either solely structural relaxation, or crystallization. After the heat treatments the $S(\Delta T)$ is illustrated for the various states of thermal history.

\footnotetext{
*corresponding author; e-mail: lovas@kgtt.bme.hu
}

\section{Results and discussion}

\subsection{Thermopower change during structural relaxation}

It has been proven, that change in the chemical composition (replacement of either the metallic host or, the type of metalloid at a fixed host metal content) result in the irreversible shift of $S(\Delta T)$. The irreversibility is the consequence of the change in the electronic structure [1]. The irreversibility (being coupled with the electron density change around the Fermi level) is common both in crystalline and in the amorphous state as well. Hence, the change in the sign and the slope of $S(\Delta T)$ is the thermodynamic manifestation of the free energy change, associated with the alloy formation. Consequently, it is also reflected in the magnitude of the enthalpy change (heat of formation) [1]. In contrast, the structural relaxation of glassy state can be either irreversible or reversible one [7]. The enthalpy change is dominantly irreversible during the relaxation [4].

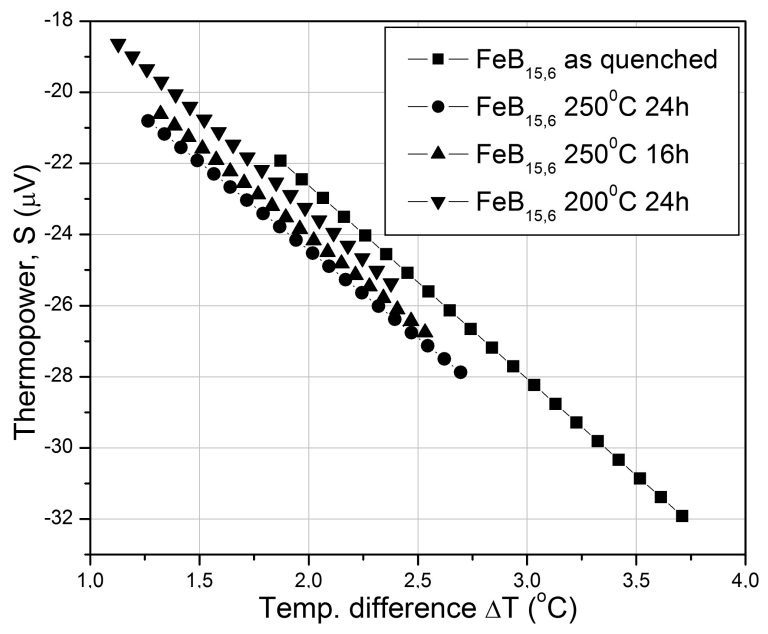

Fig. 1. The shift of thermopower during structural relaxation. 


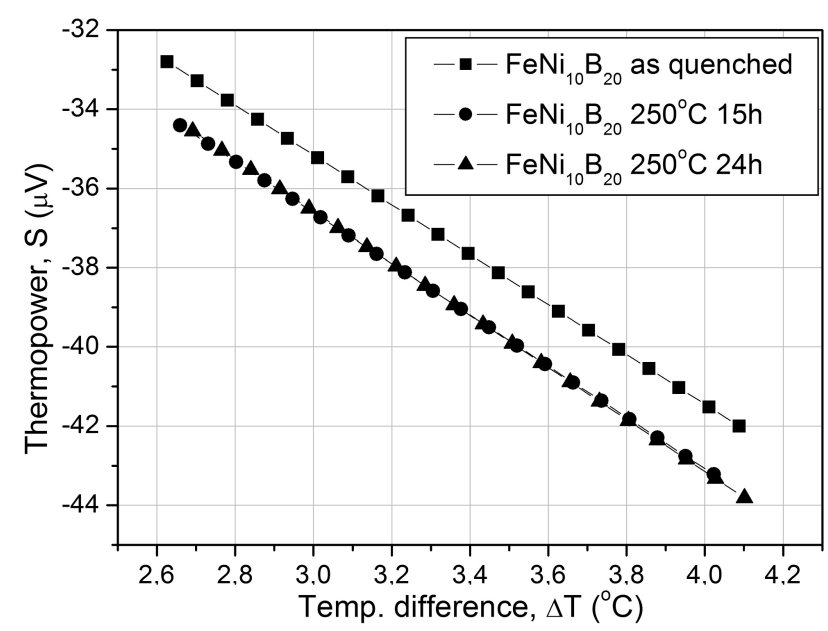

Fig. 2. The shift of $S(\Delta T)$ curves in Fe-Ni-B glass alloy during structural relaxation (isothermal heat treatments).

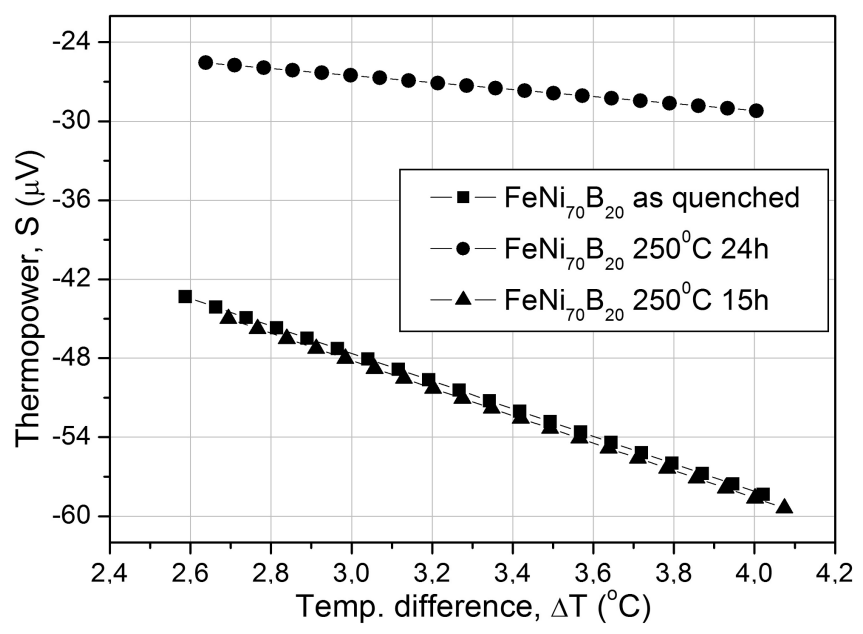

Fig. 3. $S(\Delta T)$ dependence for $\mathrm{FeNi}_{70} \mathrm{~B}_{20}$ glass in as quenched state and after the relaxation or partial crystallization.

The response of $S(\Delta T)$ to the irreversible structural relaxation is illustrated for hypo-eutectic Fe-B in Fig. 1. The $S(\Delta T)$ shifts to negative direction (compared to the as quenched state) due to the isothermal relaxation process. Similarly to the enthalpy relaxation, this shift is also irreversible. Saturation value is reached at a given temperature. Similar results are obtained in $\mathrm{Fe}-\mathrm{Ni}-\mathrm{B}$ samples (Fig. 2). The saturation trend is also obvious in this case. One can conclude therefore, that irreversible enthalpy relaxation in the glassy alloys is coupled with the irreversible $S(\Delta T)$ shift (negative direction). This effect is composition independent in the investigated glasses. On the other hand, it is important to note, that the sign of the shift is identical with that, observed in alloys with the negative heat of formation.

\subsection{Thermopower change during the amorphous-crystalline transformation}

While the glassy state is sustained during structural relaxation, phase transformation also occurs in the second case. When this process is completed, the value of thermopower depends on the crystalline phases formed during the glass decomposition. The net shape of $S(\Delta T)$ curve (sign, slope, or the absolute value at a given $\Delta T$ ) is formed via the algebraic sum of $S$, of the constituent crystalline phases i.e., the shape of curves is determined by the equilibrium mole fraction of crystalline products. This argument seems to be supported by the Fig. 3 .

In this figure, we can compare the sign of shift for $S(\Delta T)$ curves after only structural relaxation, and when a partial crystallization is already involved. The reversal of the sign, due to the beginning of crystallization is clearly visible.

\section{Conclusions}

The shape of $S(\Delta T)$ curves (sign of the shift and change in the slope) are compared in glassy $\mathrm{Fe}-(\mathrm{Ni})$-based glassy alloys after structural relaxation and partial devitrification.

1. Increase of the degree of irreversible structural relaxation, (stabilization of the bond structure) is coupled with the gradual shift of $S(\Delta T)$ into negative direction.

2. The slope of $S(\Delta T)$ is unchanged during relaxation. In contrast, the slope is significantly affected by the amorphous-crystalline transformation and the shape of $S(\Delta T)$ curve is determined by the individual, coexisting crystalline phases.

\section{References}

[1] A. Szabo, L. Novak, A. Lovas, Acta Electrotechnica et Informatica 13, 70 (2013).

[2] A. Szabó, K. Varga, A. Lovas, in: $30^{\text {th }}$ International Colloquium on Advanced Manufacturing and Repairing Technologies in Vehicle Industry, 2013, p. 29-34, ISBN 978-963-313-079-7 .

[3] L. Novák, J. Ziman, M. Kovalakova, M. Kladivová, Acta Electrotecnica et Informatica 12, 72 (2012).

[4] J.O. Ström-Olsen, R. Brüning, Z. Altounian, D.H. Ryan, Journal of the Less Common Metals 145, 327 (1988).

[5] A. Lovas, É. Kisdi-Koszó, L. Potocky, L. Novák, J. Mater. Sci. 22, 1535 (1987).

[6] M. Kladivova, J. Ziman, L. Novák, Acta Electrotechnica et Informatica 13, 65 (2013).

[7] A.L. Greer, Structural Relaxation and Atomic Transport in Amorphous Alloys in: Rapidly Solidified Alloys eds. H.H. Liebermann, M. Dekker, Inc., Basel, (1993), p. 279. 